\title{
Effects of Different Therapeutic Radiation Doses on the Development of Neural Tube Defects in Chick Embryos and the Correlation with Bone Morphogenetic Protein 4 and 7 Expression Levels
}

\author{
Eren ALTUN ${ }^{1}$, Aziz ATIK², Huseyin Utku ADILAY ${ }^{3}$, Arda KAHRAMAN ${ }^{4}$, Kerem OZTURK ${ }^{5}$, Bulent GUCLU ${ }^{6}$ \\ ${ }^{1}$ Balikesir University, School of Medicine, Department of Pathology, Balikesir, Turkey \\ ${ }^{2}$ Balikesir University, School of Medicine, Department of Orthopaedics and Traumatology, Balikesir, Turkey \\ ${ }^{3}$ Balikesir University, School of Medicine, Department of Neurosurgery, Balikesir, Turkey \\ ${ }^{4}$ Uludag University, School of Medicine, Department of Physics, Bursa, Turkey \\ ${ }^{5}$ Uludag University, School of Medicine, Department of Radiology, Bursa, Turkey \\ ${ }^{6}$ Dr. Lutfi Kirdar Training and Research Hospital, Department of Neurosurgery, Istanbul, Turkey
}

Corresponding author: Eren ALTUN erenaltun@hotmail.com

\section{ABSTRACT}

AIM: To investigate the effects of different therapeutic radiation doses on the prevalence of neural tube defects (NTDs) in chick embryos and bone morphogenetic protein (BMP) 4 and BMP7 expression levels.

MATERIAL and METHODS: The chick embryos $(n=143)$ were derived from fertile, specific pathogen-free eggs of domestic fowl. The presence of NTDs was analyzed using a stereomicroscope, and BMP4 and BMP7 expression levels were assessed by immunohistochemical staining. The chick embryos were divided into five groups: control (no radiation exposure) ( $\mathrm{n}=23)$, exposure to thorax computerized tomography $(C T)(n=30)$; exposure to abdominopelvic CT $(n=30)$, exposure to cranium CT ( $n=30)$, and exposure to brain perfusion CT ( $\mathrm{n}=30)$.

RESULTS: The prevalence of NTDs and BMP4 and BMP7 expression levels in the different groups were compared. In the cranium CT dose group, both the NTD prevalence $(20 \%, p=0.002)$ and BMP7 $(p=0.031)$ expression levels were significantly higher than those in the other groups. However, none of the medical doses of irradiation altered BMP4 expression levels ( $p=0.242$ ). No NTDs were detected in the thorax CT and abdominopelvic CT groups.

CONCLUSION: Exposure to irradiation at cranium CT doses may induce the development of NTDs and increase BMP7 expression. Dose radiation exposure using thorax CT and abdominopelvic CT protocols does not appear to induce NTDs.

KEYWORDS: Chick embryo, BMP4, BMP7, Neural tube defect, Radiation

\section{INTRODUCTION}

$\mathrm{M}$ edical imaging and therapeutic radiotherapy are the main sources of human X-ray exposure, with the aforementioned imaging modalities responsible for approximately $40 \%$ of annual exposure in the general population (13). Animal studies have proven that acute or chronic low-dose-ionizing-radiation (i.e., $\leq 100 \mathrm{mSv}$ ) and low-dose-rate-ionizing-radiation (i.e., $<6 \mathrm{mSv} / \mathrm{h}$ ) are harmful to human health. Radiation exposure may trigger genetic or epigenetic changes and cause abnormal brain growth,
Eren ALTUN $\quad$ (D): 0000-0001-9110-8364

Aziz ATIK (10): 0000-0002-2125-6465

Huseyin Utku ADILAY (1) : 0000-0003-4867-244X
Arda KAHRAMAN (1) : 0000-0003-0150-8052

Kerem OZTURK (1) : 0000-0002-6891-4613

Bulent GUCLU (1) : 0000-0002-0012-5706 
abnormal embryological development, circulatory problems, early menopause, and tumorigenesis (13). It can also have adverse effects on the immune system and longevity (13).

Bone morphogenetic proteins (BMPs) are extracellular signaling proteins that belong to the transforming growth factor beta family (5). Although they were once thought to play a role only in bone development, it is now known that they play key roles in the development of many organs and systems, especially the central nervous system (12). Oligodendrocytes and glial cells are highly sensitive to the effect of BMPs during embryonic development (12). They direct several functions, including cell proliferation, apoptosis, maturation, and migration. There are 20 structural forms of BMPs. Among these, BMP2, 4, and 7 have effects on glial cells, with most studies focusing on the effect of BMP4 on these cells (12).

During the development of the central nervous system, BMPs are found mostly in the roof plate and dorsal regions. They may also be found in ventral regions in low numbers $(8,12)$. In ventral regions, noggin, an endogenous BMP-specific inhibitor, acts as an antagonist of BMPs. In dorsal regions, BMPs exhibit a dorsalizing effect on neuronal development by enlarging the ventral sides of the neural tube (18). Previous research demonstrated in vitro developmental effects of BMPs in animal studies in which BMPs were overexpressed or inhibited. Exposure of chick embryo neural tubes to the BMP inhibitor noggin induced the formation of dorsal oligodendrocytes (9). Dorsal factors, including BMP4 and 7, induced oligodendrocyte formation in chick embryo spines by inhibiting lineage specification in vivo in middle and dorsal regions (9).

Neural tube defects (NTDs) are serious congenital malformations of the brain and spine. It is estimated that globally 300,000 newborns each year have NTDs (9). Common forms of NTDs are anencephaly and myelomeningocele, a type of spina bifida, which are caused by a closure defect in the brain or spine (7). Most NTD cases are sporadic and nonsyndromic. In terms of NTD prevention, according to previous research, periconceptional folic acid intake can reduce the prevalence of NTDs by $50-70 \%$. However, not all NTDs can be prevented by folic acid supplementation. Other factors that can prevent the development of NTDs need to be identified, and new preventive consultant strategies for NTDs need to be established (4).

The hypothesis of the present study was that different therapeutic radiation doses administered during medical investigations or treatment would alter the expression levels of BMP4 and 7, which may play a role in NTD progression.

\section{MATERIAL and METHODS}

\section{Chick Embryos and Ionized Radiation Application}

Fertile, specific pathogen-free eggs of domestic fowl (Gallus gallus domesticus; Has tavuk, Bursa, Turkey) were used in this study. The eggs were incubated at $37.5^{\circ} \mathrm{C}$ and $75 \%$ relative humidity for 24 hours until the embryos reached Hamburger and Hamilton stage six of development (6).

The eggs were divided into five groups and exposed to different types of CT radiation. In group $1(n=23)$, the eggs were not exposed to any irradiation (control group). In group $2(n=30)$, the embryos were irradiated using a thorax computerized tomography (CT) protocol. In group $3(n=30)$, abdominopelvic CT was applied. In Group 4, routine head (cranium) CT (adult dose) was applied. In group $5(\mathrm{n}=30)$, a brain perfusion CT protocol (adult dose) was used (Table I).

All CT scan protocols were calibrated using reference CT dose values recommended by the American Association of Physicists in Medicine. A 64-detector multislice CT scanner (SOMATOM Definition; Siemens Medical Solutions, Forchheim, Germany) was used. Dosimetric data and scan parameters were extracted from the Digital Imaging and Communication in Medicine Header of the CT images. An optically stimulated luminescence (OSL) dosimetery system was used to measure the doses of ionizing radiation. The system included an InLight nanoDot OSL dosimeter and a MicroStar reader (Landauer Inc., Glenwood, IL, USA). Thirty OSL nanoDot detectors were positioned at opposite poles of each of the 30 eggs in all the groups. Prior to irradiation, background radiation doses of OSL nanoDot were read using a MicroStar reader (Landauer Inc.). After irradiation by the CT scan, the OSL dosimeter was removed, and the radiation dose was read by the reader. Radiation values in dosimeters were subtracted from the final results. Irradiation dose levels were measured in miligray (mGy) with $\pm 5 \%$ tolerance.

\section{Embryo Collection}

After irradiation, the eggs were placed in an incubator for 24 hours until developmental stage 12. At the end of incubation (i.e., 48 hours from the onset of the experiment), the eggs were cracked open. After dissection of the allantoic stalk from other embryonic structures, the embryos were transferred to a petri dish.

Table I: Chicken Egg Groups and Their CT Radiation Protocols

\begin{tabular}{lcl}
\hline Group & n (Eggs) & Radiation Dose \\
\hline Control & 23 & Not exposed to radiation \\
\hline Chest CT & 30 & Irridiated using lung cancer screening chest CT protocol \\
\hline Abdominopelvic CT & 30 & Irridiated using adult routine abdominopelvic CT protocol \\
\hline Brain CT & 30 & Irridiated using adult routine head CT protocol \\
\hline Brain Perfusion CT & 30 & Irridiated using adult brain perfusion CT protocols \\
\hline
\end{tabular}




\section{Examination Under a Stereomicroscope and Analysis of BMP4 and BMP7 Expression by Immunohistochemistry}

Tap water was added to the embryos in the petri dish, and they were examined under a stereomicroscope (Olympus, SZX/SZ, Olympus Corporation, Japan) by a pathologist blinded to the protocols. Neural tube closure and neural tube developmental abnormalities were recorded.

The specimens were kept in $10 \%$ formalin solution for 48 hours prior to a histopathological assessment. In accordance with standard practice, the specimens were passed through an alcohol (70\%, 80\%, 90\%, 96\%, and 100\%) and xylol series and then embedded in paraffin blocks. Each block was sliced into $4 \mu \mathrm{m}$-thick sections and treated with BMP4 and BMP7 and the following primary antibodies (BMP4: 1:100, rabbit, polyclonal, Biorbyt LLC., San Francisco, CA, USA; BMP7: 1:100, rabbit, polyclonal, Biorbyt LLC). Immunohistochemical staining was performed using a Ventana Benchmark XT autostainer with an XT ultraView DAB kit (Ventana Medical Systems, Roche Diagnostics Co., Mannheim, Germany). Cytoplasmic and nuclear staining were accepted as positive for cellular immunohistochemical expression. A pathologist blinded to the study protocol assessed the clinical findings using a light microscope. (Nikon Eclipse Cl; Nikon, Amsterdam, the Netherlands). For automatic scoring, the histogram profile of each image, which indicated the pixel count to a specific intensity level, was evaluated using the program Nis Elements 4.30 (Nikon, Amsterdam, the Netherlands). Pixel density levels for an unspecific stain were excluded. For all tissues, the intensity level was determined after manual selection of the area by the pathologist.

\section{Statistical Analysis}

The conformity of the data (dose amount, BMP4 level, and BMP7 level) to a normal distribution was investigated using the Shapiro-Wilk test. Related variables were expressed as median (minimum: maximum) according to the test results. The Kruskal-Wallis test was used to compare BMP4 and BMP7 levels in the various groups. For binary comparisons between groups, the Dunn-Bonferroni method was used. The Mann-Whitney $U$ test was used to compare NTDs and BMP4 and BMP7 expression levels in the different radiation dose groups as compared with those in the control group. The NTD variable was expressed as numbers and percentages, and intergroup comparisons of NTDs were investigated using the Fisher-Freeman-Halton test. For subgroup analysis, Fisher's exact chi-square test was used. All statistical analyses were performed using IBM SPSS Statistics for Windows, version 21.0 (IBM Corp., Armonk, NY, USA). The accepted significance level was $\mathrm{a}=0.05$.

\section{Ethics Committee Approval}

All the experimental procedures were reviewed and approved by the animal research ethics committee of Balikesir University (Decision No: 2018/8-7). Animal care and all experiments adhered to European Communities Council Directive (86/609/ EEC) on the protection of animals for experimental use.

\section{RESULTS}

Table II provides data on NTDs and BMP4 and BMP7 expression levels in the different radiation dose groups as compared with those in the control group. No NTDs were detected in the thorax CT and abdominopelvic CT groups. NTDs were detected in 1 (3.3\%) chick embryo in the perfusion CT group, and 6 (20\%) chick embryos in the cranium CT group (Figure 1A, B). In the subgroup analysis, the prevalence of NTDs in the cranium CT group was higher than that in the abdominal CT and thorax CT groups $(p=0.002)$.

There was no statistically significant between-group difference in BMP7 levels. In the subgroup analysis, the median BMP7 expression level in the cranium CT group was higher than that in the abdominal CT, thorax CT, and control groups $(p=0.001)$ (Figure 2A, B; Table III).

There was no statistically significant between-group difference in BMP4 levels $(p=0.242)$.

\section{DISCUSSION}

This study investigated the effect of different therapeutic radiation doses on chick embryos in terms of the prevalence of NTDs as assessed by a stereomicroscope study and BMP4 and BMP7 expression levels, which were determined by an immunohistochemical investigation. In the cranium CT group, both the NTD prevalence and BMP7 expression level were

Table II: The Details of CT Radiation and the Presence of Neural Tube Defects and the Results of the Expression of BMP4 and BMP7

\begin{tabular}{|c|c|c|c|c|}
\hline & $\begin{array}{c}\text { Dose } \\
\text { median (min-max.) }\end{array}$ & $\begin{array}{l}\text { NTD } \\
\text { n (\%) }\end{array}$ & $\begin{array}{c}\text { BMP7 } \\
\text { median (min-max.) }\end{array}$ & $\begin{array}{c}\text { BMP4 } \\
\text { median (min-max.) }\end{array}$ \\
\hline Control & 0 & $0(0)$ & $171.50(152-202)$ & $133(80-190)$ \\
\hline Chest CT & $1.10(0.61-1.55)$ & $0(0)$ & $166(147-187)$ & $135.50(83-182)$ \\
\hline Abdominopelvic CT & $22.95(17-31)$ & $0(0)$ & $168.50(143-210)$ & $128(90-202)$ \\
\hline Cranium CBT & $47.04(35.41-58.98)$ & $6(20)$ & $203(124-218)$ & $132(91-205)$ \\
\hline Brain Perfusion CT & $321.45(233.30-571.26)$ & $1(3.3)$ & $183(159-215)$ & $144.50(121-194)$ \\
\hline $\mathbf{p}$ & - & $0.002^{a}$ & $<0.001^{b}$ & $0.242^{b}$ \\
\hline
\end{tabular}

a: Fisher-Freeman-Halton Test, b: Kruskal Wallis Test. 

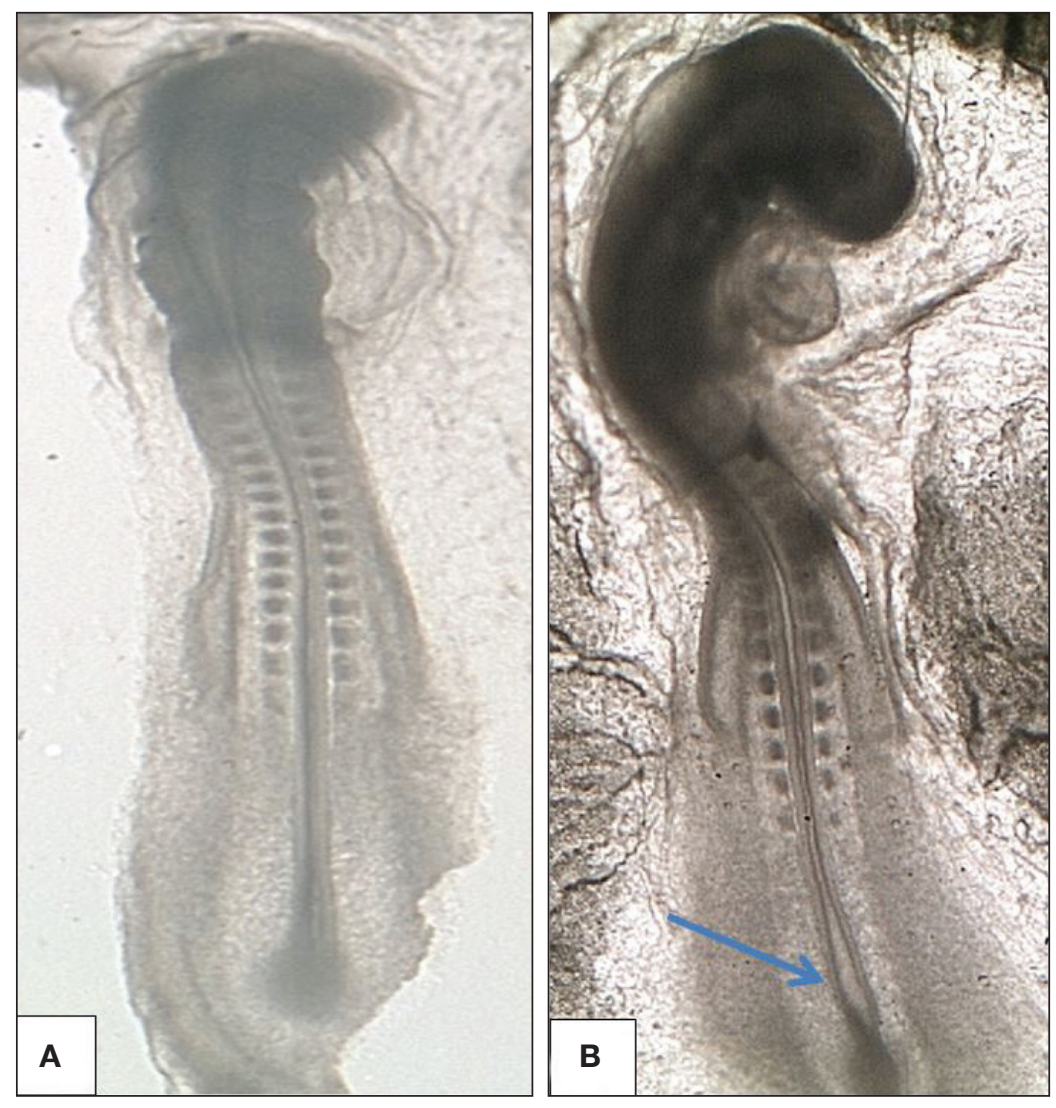

Figure 1: A) Closed neural tube of a normal chick embryo in control group. B) Neural tube abnormality (pointed by arrow).
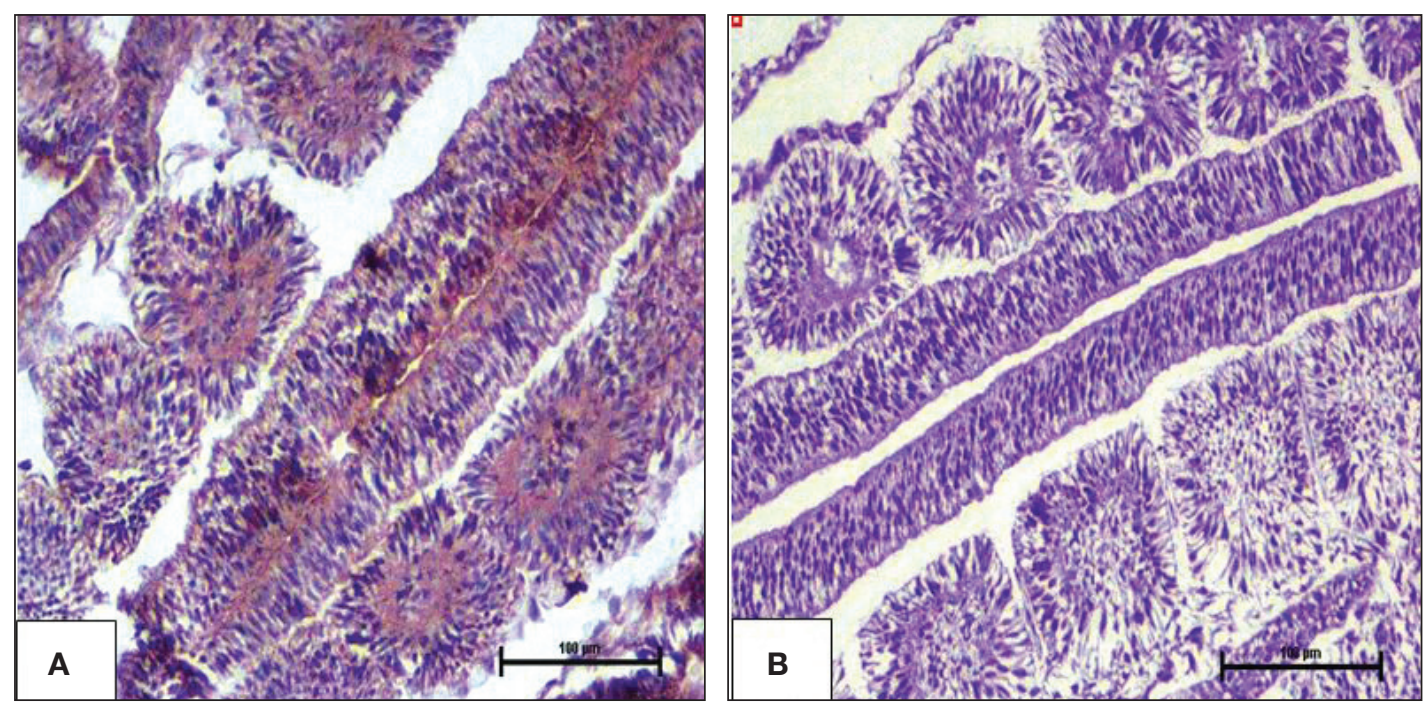

Figure 2: A) The expression of BMP7; chicken embryo cells showing distinct cytoplasmic apical immunoreactivity (x100). B) The chicken embryo cells showing no nuclear immunoreactivity of BMP7 (x100).

significantly higher as compared with the same parameters in the other groups. The medical doses of irradiation administered did not alter BMP4 expression levels. No NTDs were detected in the thorax CT and abdominopelvic CT groups.

Since their first discovery as osteoinductive factors, BMPs have been shown to affect the neurological system, especially in terms of neuroectoderm induction, neural crest cell specification, and neuronal arrangement in the central nervous system (3). BMP ligands attach to serine/threoninekinase transmembrane receptors and initiate phosphorylation of signal induction molecules SMAD1, SMAD5 and SMAD8 (10). These phosphorylated SMADs bind to SMAD4 and penetrate the cell nucleus to regulate target gene transcription $(1,10)$.

BMP4 plays a critical role in early murine embryological development, with deletion of BMP4 proving lethal 6.59.5 days postcoitum $(19,20)$. BMP expression in early 
Table III: NTD Levels according to BMP7 Expression, Subgroup Analysis

\begin{tabular}{|c|c|c|c|}
\hline \multirow[b]{2}{*}{$\left(p_{\text {group i v.s. group j }}\right)$} & \multicolumn{3}{|c|}{ Pairwise Comparisons } \\
\hline & Dose & NTD & BMP7 \\
\hline $\mathbf{P}_{\text {Abdominal } C T \text { - Thorax CT }}$ & - & 1.00 & 1.00 \\
\hline $\mathbf{P}_{\text {Abdominal } C T \text { - Control }}$ & $<0.001$ & 1.00 & 1.00 \\
\hline $\mathbf{P}_{\text {Abdominal } C T-\text { Cranium } C T}$ & - & 0.027 & 0.001 \\
\hline $\mathbf{P}_{\text {Abdominal } C T \text { - Perfusion } C T}$ & - & 0.463 & 0.138 \\
\hline $\mathbf{P}_{\text {Thorax } C T \text { - Control }}$ & $<0.001$ & 1.00 & 1.00 \\
\hline $\mathbf{P}_{\text {Thorax } C T \text { - Cranium } C T}$ & - & 0.023 & $<0.001$ \\
\hline $\mathbf{P}_{\text {Thorax } C T \text {-Perfusion } C T}$ & - & 0.422 & 0.068 \\
\hline $\mathbf{P}_{\text {Control-Cranium } C T}$ & $<0.001$ & 0.029 & 0.031 \\
\hline $\mathbf{P}_{\text {Control - Perfusion } C T}$ & $<0.001$ & 0.475 & 0.861 \\
\hline $\mathbf{P}_{\text {Cranium-Perfusion } C T}$ & - & 0.213 & 1.00 \\
\hline
\end{tabular}

embryological development is actively inhibited by the secretion of embryonic factors, including noggin, chordin, and follistatin, to initiate neural induction (3). In vitro treatment of human embryonic stem cells with noggin induces degradation of SMAD4 transcripts via microRNA. This mechanism blocks the BMP4-SMAD signal pathway during neural induction in vivo. Repression or activation of BMP signaling, in conjunction with a corresponding gradient of sonic Hedgehog (shh) expression, specifies whether ectoderm gives rise to neuronal or nonneuronal tissue (3). In neuronal differentiation of chick embryos, fibroblast growth factor 3 (FGF3), BMP4, and BMP7 are expressed in cells destined to become neural cells. FGF signaling is essential for inhibiting BMP expression and for neural cell development. Besides, later inhibition of BMP on epiblast cells may inhibit neural tissue development and induce epidermal-characterized cells (17). BMP7 and FGF8 are expressed in the branchial arches in chick embryos. BMP7 and FGF8 are expressed in the posterior ectodermal margin of the second arch. Thus, inhibitor signals of BMPs influence the activity of FGFs (16). During chick gastrulation, BMP2, 4, and 7 are strongly expressed in the neural plate border, whereas phosphorylated SMAD1, 5, and 8 are moderately expressed in the neural plate border $(2,11)$.

Recent research showed that de novo mutations were a common cause of neurodevelopmental malformations, such as NTDs (14). According to previous research, the prevalence of NTDs was $0.1-0.2 \%(7)$.

\section{CONCLUSIONS}

lonizing radiation is widespread, with potential sources including human-made sources, such as industry, especially the medical industry, and naturally occurring sources (radon and cosmic). Cumulative exposure as a result of repetitive exposure to low-dose medical radiation, such as via CT, may exceed permissible limits (15). Radiation exposure through inappropriate use of diagnostic modalities is a cause of concern. We believe that the low-dose effects of irradiation, especially in the case of pregnant women and embryos, should be investigated in detail. In our study, there was no significant difference in the BMP4 expression level in any of the groups, irrespective of the irradiation dose. However, in the cranium CT group, BMP7 levels were significantly higher, with a significant increase in NTD development. Our results suggest that exposure to irradiation at cranium CT doses may induce the development of NTDs via increased expression of BMP7.

\section{ACKNOWLEDGEMENTS}

This research is funded by Balıkesir University (BAP. 2017/203).

\section{- REFERENCES}

1. Bier E, De Robertis EM: BMP gradients: A paradigm for morphogen-mediated developmental patterning. Science 348(6242):aaa5838, 2015

2. Chapman SC, Schubert FR, Schoenwolf GC, Lumsden A: Analysis of spatial and temporal gene expression patterns in blastula and gastrula stage chick embryos. Dev Biol 245: 187-199, 2002

3. Cole AE, Murray SS, Xiao J: Bone morphogenetic protein 4 signalling in neural stem and progenitor cells during development and after injury. Stem Cells Int 2016:1-16, 2016

4. Copp AJ, Stanier P, Greene NDE: Neural tube defects: Recent advances, unsolved questions, and controversies. Lancet Neurol 12:799-810, 2013

5. Grinspan JB: Bone morphogenetic proteins: Inhibitors of myelination in development and disease. Bone Morphog Protein 99:195-222, 2015

6. Hamburger V, Hamilton HL: A series of normal stages in the development of the chick embryo. J Morphol 88:49-92, 1951

7. Lemay P, Guyot MC, Tremblay É, Dionne-Laporte A, Spiegelman D, Henrion É, Diallo O, De Marco P, Merello E, Massicotte C, Désilets V, Michaud JL, Rouleau GA, Capra V, Kibar Z: Loss-of-function de novo mutations play an important role in severe human neural tube defects. J Med Genet 52(7): 493-497, 2015

8. Liem KF, Tremml G, Roelink H, Jessell TM: Dorsal differentiation of neural plate cells induced by BMP-mediated signals from epidermal ectoderm. Cell 82:969-979, 1995

9. Mekki-Dauriac S, Agius E, Kan P, Cochard P: Bone morphogenetic proteins negatively control oligodendrocyte precursor specification in the chick spinal cord. Development 129:5117-5130, 2002

10. Shi Y, Massagué J: Mechanisms of TGF- $\beta$ signaling from cell membrane to the nucleus. Cell 113:685-700, 2003

11. Stuhlmiller TJ, García-Castro MI: FGF/MAPK signaling is required in the gastrula epiblast for avian neural crest induction. Development 139:289-300, 2012

12. Sussel L, Marin O, Kimura S, Rubenstein JL: Loss of Nkx2.1 homeobox gene function results in a ventral to dorsal molecular respecification within the basal telencephalon: Evidence for a transformation of the pallidum into the striatum. Development 126(15):3359-3370, 1999 
13. Tang FR, Loke WK, Khoo BC: Low-dose or low-dose-rate ionizing radiation-induced bioeffects in animal models. J Radiat Res 58:165-182, 2017

14. Veltman JA, Brunner HG: De novo mutations in human genetic disease. Nat Rev Genet 13:565-575, 2012

15. Verreet T, Verslegers $M$, Quintens R, Baatout S, Benotmane MA: Current evidence for developmental, structural, and functional brain defects following prenatal radiation exposure. Neural Plast 2016:1243527, 2016

16. Wall NA, Hogan BLM, Walb NA, Hogawb BLM: Expression of bone morphogenetic protein-4 (BMP-4), bone morphogenetic protein-7 (BMP-7), fibroblast growth factor-8 (FGF-8) and sonic hedgehog $(\mathrm{SHH})$ during branchial arch development in the chick. Mech Dev 53:383-392, 1995
17. Wilson SI, Graziano E, Harland R, Jessell TM, Edlund T: An early requirement for FGF signalling in the acquisition of neural cell fate in the chick embryo. Current Biology 10:421429, 2000

18. Wine-Lee L, Ahn KJ, Richardson RD, Mishina Y, Lyons KM, Crenshaw EB 3rd: Signaling through BMP type 1 receptors is required for development of interneuron cell types in the dorsal spinal cord. Development 131:5393-5403, 2004

19. Winnier G, Blessing M, Labosky PA, Hogan BL: Bone morphogenetic protein-4 is required for mesoderm formation and patterning in the mouse. Genes Dev 9:2105-2116, 1995

20. Zaganjor I, Sekkarie A, Tsang BL, Williams J, Razzaghi H, Mulinare J, Sniezek JE, Cannon MJ, Rosenthal J: Describing the prevalence of neural tube defects worldwide: A systematic literature review. PLoS One 11(4):e0151586, 2016 